\title{
A Distinct Set of Drosophila Brain Neurons Required for Neurofibromatosis Type 1-Dependent Learning and Memory
}

\author{
Monica E. Buchanan ${ }^{1}$ and Ronald L. Davis ${ }^{1,2}$ \\ Department of ${ }^{1}$ Molecular and Human Genetics and ${ }^{2}$ Molecular and Cellular Biology, Baylor College of Medicine, Houston, Texas 77030
}

\begin{abstract}
Nonspecific cognitive impairments are one of the many manifestations of neurofibromatosis type 1 (NF1). A learning phenotype is also present in Drosophila melanogaster that lack a functional neurofibromin gene ( $n f 1)$. Multiple studies have indicated that Nf1-dependent learning in Drosophila involves the cAMP pathway, including the demonstration of a genetic interaction between Nf1 and the rutabagaencoded adenylyl cyclase (Rut-AC). Olfactory classical conditioning experiments have previously demonstrated a requirement for Rut-AC activity and downstream cAMP pathway signaling in neurons of the mushroom bodies. However, Nf1 expression in adult mushroom body neurons has not been observed. Here, we address this discrepancy by demonstrating (1) that Rut-AC is required for the acquisition and stability of olfactory memories, whereas Nf1 is only required for acquisition, (2) that expression of $n f 1$ RNA can be detected in the cell bodies of mushroom body neurons, and (3) that expression of an $n f 1$ transgene only in the $\alpha / \beta$ subset of mushroom body neurons is sufficient to restore both protein synthesis-independent and protein synthesis-dependent memory. Our observations indicate that memory-related functions of Rut-AC are both Nf1-dependent and -independent, that Nf1 mediates the formation of two distinct memory components within a single neuron population, and that our understanding of Nf1 function in memory processes may be dissected from its role in other brain functions by specifically studying the $\alpha / \beta$ mushroom body neurons.
\end{abstract}

\section{Introduction}

Neurofibromatosis type 1 (NF1) is an autosomal, dominant genetic disorder that afflicts approximately 1 in every 3500 individuals. Like other clinical manifestations of NF1, expression and penetrance of cognitive phenotypes varies and may include deficiencies of visual-spatial processing, executive function, and attention (for review, see Ozonoff, 1999; North, 2000; Acosta et al., 2006). Homologs of human Nf1 in mouse and Drosophila melanogaster share significant identity at the protein level, and animal models in both species were developed shortly after the human Nf1 gene was cloned (Bernards et al., 1993; Jacks et al., 1994; The et al., 1997). Both models demonstrate cognitive phenotypes, and insights gained through animal studies have shed light on the genetic and biochemical basis of these defects.

Drosophila has been used extensively for expanding our basic understanding of memory, making it ideal for investigating NF1 cognitive deficits. After olfactory classical conditioning, Drosophila form protein synthesis-independent early memories (PSIEM), comprising short-term memory (STM) tested at 3 min after training, middle-term memory often tested at $3 \mathrm{~h}$ after training, and protein synthesis-dependent long-term memory (PSDLTM), tested at $24 \mathrm{~h}$ after conditioning. The $n f 1$ mutant flies demonstrate deficiencies in PSI-EM and PSD-LTM (Guo et al.,

Received Jan. 18, 2010; revised Feb. 16, 2010; accepted Feb. 17, 2010.

These studies were supported by National Institutes of Health Grant NS19904 (R.L.D.). M.E.B. was supported by a Children's Tumor Foundation Young Investigator Award and National Institute of General Medical Sciences Grant T32GM008307. We thank Curtis Wilson for technical assistance.

Correspondence should be addressed to Ronald L. Davis at his present address: Department of Neuroscience, The Scripps Research Institute, Jupiter, FL 33458. E-mail: rdavis@scripps.edu.

DOI:10.1523/JNEUROSCI.0283-10.2010

Copyright $\odot 2010$ the authors $\quad$ 0270-6474/10/3010135-09\$15.00/0
2000; Ho et al., 2007). A current model postulates that Nf1 contributes to PSI-EM through stimulation of the rutabaga-encoded adenylyl cyclase (Rut-AC) (Guo et al., 2000). Stimulation of $\mathrm{G} \alpha_{\mathrm{s}}$ dependent $\mathrm{AC}$ activity requires only the Nf1 C-terminal domain (Hannan et al., 2006). The PSI-EM phenotypes of $n f 1$, rut- $A C$, and $n f 1 /$ rut- $A C$ mutants are similar (Guo et al., 2000), both genes are required at the time of learning (Guo et al., 2000; McGuire et al., 2003; Mao et al., 2004), and either ubiquitous expression of a constitutively active protein kinase $\mathrm{A}\left(h s P K A^{*}\right)$ transgene (Guo et al., 2000) or neuronal expression of a Nf1 C-terminal domain transgene (Ho et al., 2007) rescues the $n f 1$ phenotype. Furthermore, the current model also postulates that Nf1 contributes to PSD-LTM through regulation of Ras via its GAP-related domain (GRD) (Hannan et al., 2006; Ho et al., 2007). Stimulation of Ras-dependent AC activity is absent in nfl mutants, but transgenic expression of the Nf1 GRD restores this activity (Hannan et al., 2006) and improves the PSD-LTM phenotype of $n f 1$ mutants (Ho et al., 2007).

It is surprising that endogenous Nf1 expression has not been observed in adult mushroom body (MB) neurons (Walker et al., 2006). MB neurons are essential for olfactory memory formation (McGuire et al., 2003; Davis, 2005), and Rut-AC is preferentially expressed in these neurons (Han et al., 1992). Rescue experiments demonstrated that transgenic expression of $r u t-A C$ in $\alpha / \beta$ and $\gamma \mathrm{MB}$ neurons restores normal memory in homozygous mutants (Zars et al., 2000; McGuire et al., 2003; Mao et al., 2004; Akalal et al., 2006; Blum et al., 2009). If Nf1 indeed stimulates Rut- $\mathrm{AC}$ activity during learning, it is probably expressed, and required, in $\mathrm{MB}$ neurons.

Here, we explore whether $\mathrm{Nf1}$ and Rut-AC are involved in the same operational phase of learning, whether they are expressed in 
the same neurons, whether both are required in the same neurons for rescue of PSI-EM, and whether the Ras-mediated function of $\mathrm{Nf1}$ is required in overlapping neurons. We report a role for Rut-AC in memory stability that is Nf1 independent, observe $n f 1$ expression in $\mathrm{MB}$ neurons, and demonstrate a requirement for $n f 1$ expression in $\alpha / \beta \mathrm{MB}$ neurons for both PSI-EM and PSD-LTM.

\section{Materials and Methods}

Fly culture and genetics. Flies were reared on standard cornmeal medium, at $25^{\circ} \mathrm{C}, 60 \%$ relative humidity, and a $12 \mathrm{~h}$ light/dark cycle. For Gene-Switch experiments, an appropriate volume of RU486 (11 $\beta$-[p(dimethylamino)phenyl]-17 $\beta$-hydroxy-17-(1-propynyl)estra-4,9-dien3 -one) stock solution was mixed into molten standard medium at $65^{\circ} \mathrm{C}$, along with food coloring, to a final concentration of $200 \mu \mathrm{M}$. Flies were reared and maintained on this altered food source throughout development and adulthood or were transferred from standard food to food containing RU486 at eclosion and were trained at $5 \mathrm{~d}$ after eclosion. A. Bernards (Massachusetts General Hospital, Boston, MA), A. Sehgal (University of Pennsylvania, Philadelphia, PA), and M. Stern (Rice University, Houston, TX) provided fly stocks. All stocks used in this study were outcrossed to our $w(C S 10)$ stock ( $w^{1118}$ flies outcrossed to Canton-S for 10 generations) for six generations, except for the $r u t^{2080}$ stock, which contains a P-element insertion bearing the rosy gene. As a result, rut ${ }^{2080}$ is maintained in a ry background. K33 flies contain a P-element in the $E(s p l)$ complex, which was mobilized to produce the $n f 1^{P 2}$ allele. Subsequently, an imprecise excision of the $n f 1^{P 2}$ P-element produced the $n f 1^{P 1}$ allele (The et al., 1997). K33 flies do not show a behavioral phenotype relative to $w$ (CS10) flies, so both of these lines were used interchangeably as controls (labeled as "control" in figures). PBac $\{\mathrm{PB}\} \mathrm{Nf1} 1^{\mathrm{c} 00617}$ $\left(n 1^{\text {c00617 }}\right.$ ) flies (Thibault et al., 2004) were obtained from the Bloomington Drosophila Stock Center (Bloomington, IN). We confirmed the insertion site of $n f 1^{100617}$ via inverse PCR using primer sequences generated and made publicly available by Exelixis through the Bloomington Drosophila Stock Center. As reported on the FlyBase website, the stock $n f 1^{\text {co0617 }}$ contains an insertion of the $\mathrm{PBac}\{\mathrm{PB}\}$ transposon in the seventh intron of the $n f 1$ genomic sequence, at position 21811820 of the D. melanogaster genome (R5.5).

Behavioral assays. Olfactory learning and memory experiments were conducted using an olfactory classical conditioning paradigm (Beck et al., 2000). Standard training procedures were used for all $3 \mathrm{~min}$ and $3 \mathrm{~h}$ memory experiments. Two- to 7-d-old flies were exposed to a single training trial, in which they were sequentially presented with methylcyclohexanol (MCH) and benzaldehyde (BA) odors for 1 min each. During presentation of the first odor [conditioning stimulus $(\mathrm{CS}+)$ ], flies were simultaneously exposed to $12-1.25 \mathrm{~s}$ pulses of $90 \mathrm{~V}$ electric shock. After a 30 s delay, the second odor (CS-) was presented in the absence of electric shock. Flies were then transferred to a T-maze and allowed to choose between the two trained odors, each contained within one arm of the maze. Avoidance of the CS+ during testing was calculated as a performance index (P.I.), defined as the fraction of flies preferring the CSminus the fraction of flies preferring the CS + . A P.I. of 1.0 indicates that all flies avoided the CS+, and a P.I. of 0.0 indicates a 50:50 distribution between the T-maze arms and therefore no learning. To control for possible naive odor bias, each trial comprised two groups in which the first group was trained with $\mathrm{MCH}$ as the $\mathrm{CS}+$, and the second group was trained with $\mathrm{BA}$ as the $\mathrm{CS}+$. To control for visual distraction, all experiments were performed in a darkroom illuminated with dark red light. Environment within the darkroom was also controlled at $23-25^{\circ} \mathrm{C}$ and $65-75 \%$ humidity. For $24 \mathrm{~h}$ memory assay, flies were 1-to 4 -d-old, and either a $5 \times$-massed or $5 \times$-spaced protocol was followed. During massed training, flies were presented with five training cycles, as described above, with a 30 s intertrial interval. During spaced training, flies were presented with five training cycles with a 15 min intertrial interval, to elicit PSDLTM (Yu et al., 2006). For memory acquisition and stability assays, a modified training paradigm known as the short program was used (Beck et al., 2000). In this schedule, odor exposures were reduced to $10 \mathrm{~s}$ with a single 1.25 s electric shock presented at the eighth second and a 30 s delay between odor presentations. When multiple training trials were presented, there was a $30 \mathrm{~s}$ intertrial interval.

RNA in situ hybridization. Probe template containing an 875 bp region, including exons 6 and 7, was amplified from NF1 mini plasmid (A. Bernards) by PCR and cloned into pCRII-TOPO plasmid (Invitrogen). Digoxigenin (DIG)-labeled RNA probes were transcribed from linearized plasmid in the antisense orientation, using the DIG RNA Labeling kit (SP6/T7) (Roche). Both $w(C S 10)$ and $n f 1^{P 1}$ fly heads were cryosectioned $(15 \mu \mathrm{m})$ and fixed in $4 \%$ paraformaldehyde. Sections were denatured with $0.2 \mathrm{~N} \mathrm{HCl}$, treated with Proteinase $\mathrm{K}$, postfixed in $4 \%$ paraformaldehyde, and acetylated. After a $1 \mathrm{~h}$ prehybridization at $50^{\circ} \mathrm{C}$, denatured DIG-labeled probes were hybridized to sections at $50^{\circ} \mathrm{C}$ for 16-24 h. Hybridization buffer contained 50\% formamide, $5 \times$ SSC, $5 \times$ Denhardt's solution, $250 \mu \mathrm{g} / \mathrm{ml}$ yeast tRNA, $500 \mu \mathrm{g} / \mathrm{ml}$ salmon sperm DNA, $50 \mu \mathrm{g} / \mathrm{ml}$ heparin, $2.5 \mathrm{~mm}$ EDTA, 0.1\% Tween 20, and $0.25 \%$ 3-[(3-cholamidopropyl)dimethylammonio]-1-propanesulfonate. After a number of washes to decrease salt concentration, slides were incubated with $\alpha$-DIG-alkaline phosphate (Roche) and visualized using nitrobluetetrazolium-chloride/5-bromo-4-chlor-indolyl-phosphate (Roche) solution. When staining was complete, slides were washed in PBST (PBS and Triton X-100) and mounted in Glycergel (Dako).

\section{Results}

\section{$n f 1^{\text {c00617 }}$ mutants exhibit size and cognitive phenotypes}

We first explored whether the putative allele $n f 1^{\text {coo617 }}$ conferred the small size and learning phenotypes typical of $n f 1^{P 1}$ and $n f 1^{P 2}$ mutants (The et al., 1997; Guo et al., 2000). To measure body size, female and male adult flies were measured from the anterior tip of the antennae to the posterior tip of the abdomen. As expected, both $n f 1^{P 1}$ and $n f 1^{P 2}$ males and females were significantly smaller than control males and females, respectively (The et al., 1997). In contrast, only $n f 1^{c 00617}$ males were smaller than control males, but the $n f 1^{\text {c00617 }}$ males were still significantly larger than $n f 1^{P 1}$ and $n f 1^{P 2}$ males. There was no significant size difference between $n \mathrm{nf}^{\mathrm{c00617}}$ and control females (Fig. 1A).

In addition to a mild size phenotype, $n f 1^{\text {c00617 }}$ homozygous mutant flies also exhibited $3 \mathrm{~min}, 3 \mathrm{~h}$, and $24 \mathrm{~h}$ memory phenotypes (Fig. $1 B$ ). nf1 $1^{\text {c00617 }}$ performance at all time points was significantly poorer than the control, but, when tested $3 \mathrm{~min}$ after training, these flies performed significantly better than $n f 1^{P 1}$, $n \mathrm{fl}^{\mathrm{P2}}$, and $r u \mathrm{t}^{2080}$ homozygous mutants. We also sought to confirm that the cognitive phenotype of $n f 1^{c 00617}$ mutants is attributable to a disruption of Nf1 function. We expressed a transgene containing full-length Drosophila nf1 (uas-dnf1) in the $n f 1^{\text {coo617 }}$ homozygous mutant background using the elav-gal4 driver, which promotes gene expression in all neurons. Restoration of $n f 1$ expression in all neurons fully rescued the $3 \mathrm{~h}$ memory phenotype of $n f 1^{\text {coo617 }}$ homozygous mutants, confirming that the $n f 1^{c 00617}$ insertion does indeed disrupt Nf1-dependent memory (Fig. 1C).

\section{$n f 1$ mutants are defective in memory acquisition but not memory stability}

Although a requirement for neurofibromin during olfactory conditioning was established previously (Guo et al., 2000), former experiments did not address which operational phase of learning is impaired. Any deficit in olfactory memory may represent a failure to associate the odor and shock stimuli, an increased rate of memory decay, or failure of memory retrieval (Cheng et al., 2001). Memory acquisition and stability were assayed for $n f 1^{100617}, n \mathrm{fl}^{P 2}$, and $r u t^{2080}$ homozygous null mutants. Flies were presented $1-15$ training trials with a $30 \mathrm{~s}$ intertrial interval, and performance was assayed immediately after the last training trial (Fig. 2A). Control performance improved with increasing number of training trials and reached a plateau at a 

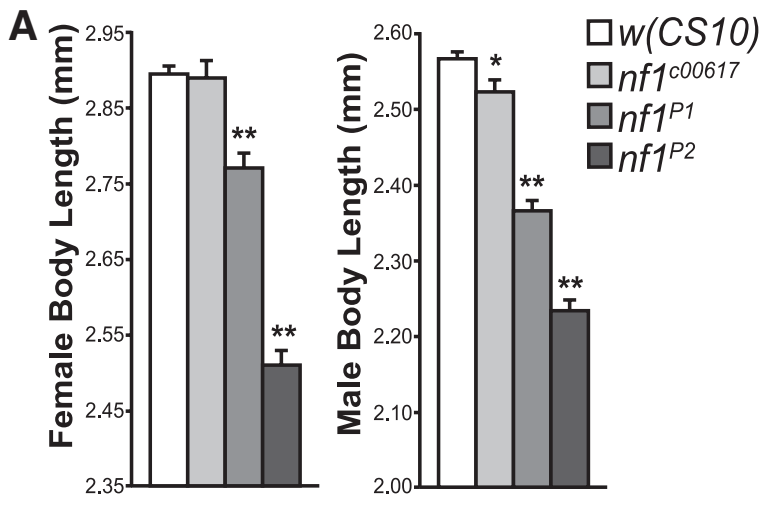

B

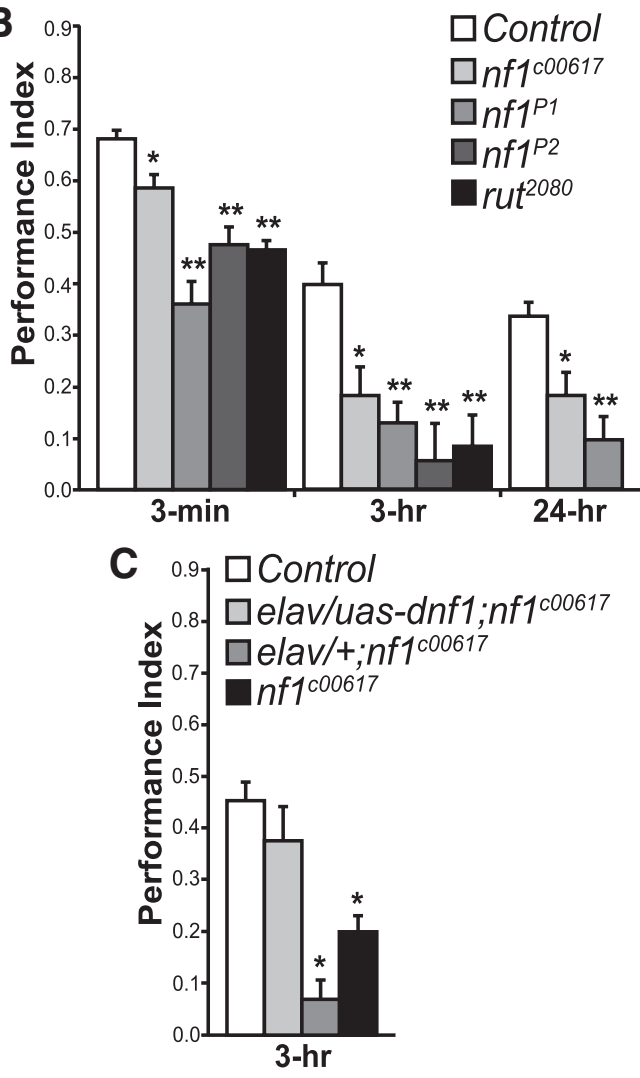

Figure 1. $n f 1$ mutants exhibit size and memory phenotypes. $A$, Female and male adults were measured from the anterior tip of the antennae to the posterior tip of the abdomen. There was no significant difference between $n f f^{c 00617}$ and control females, but $n f f^{\text {c00617 }}$ males were significantly smaller than control males $\left({ }^{*} p<0.005\right)$. Both genders of $n f f^{P 2}$ and $n f f^{P 1}$ were significantly smaller than both controls and $n f 1^{\text {c00617 }}$, and $n f f^{P 2}$ flies were also significantly smaller than $n f 1^{P 1}$. For control, $w(C S 10)$ was used, $n=100$, and for all $n f 1$ mutants, $n=50$. Means \pm SEM are shown. ${ }^{*} p<0.005,{ }^{* *} p<0.0001 . B$, Learning and memory phenotype of $n f 1^{\text {c00617 }}$ homozygous mutants compared with other $n f 1$ alleles. Performance was assayed 3 $\mathrm{min}, 3 \mathrm{~h}$, and $24 \mathrm{~h}$ after training. All mutant groups showed impaired performance relative to controls. $n f 1^{600617}$ mutants performed significantly better than $n f 1^{P 1}, \mathrm{nf}^{\mathrm{P2}}$, and $\mathrm{rut}^{2080} \mathrm{mu}-$ tants when tested at $3 \mathrm{~min}$ after training but not when tested 3 or $24 \mathrm{~h}$ after training. A $5 \times$-spaced protocol was used to elicit $24 \mathrm{~h}$ memory. For 3 min and $3 \mathrm{~h}$ memory, $w(\mathrm{CS} 10)$ was used as a control. K33 was used as a control for $24 \mathrm{~h}$ memory. C, Expression of $n f 1$ in all neurons rescues the $3 \mathrm{~h}$ memory phenotype of $n f 1^{\text {c00617 }}$ mutants. Both control and elav/uas-dnf1; $n f 7^{100617}$ flies performed significantly better than both elav/+; $n f 7^{00617}$ and $n f f^{\text {c00617 }}$ flies,

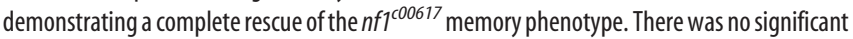
difference between the performance of control and elav/uas- dnf1,nf $1^{\text {c00617 }}$ flies or between elav/+; $n f^{100617}$ and $n f 1^{000617}$ flies. There was also no significant difference between the performance of $n f^{100617}$ and uas-dnf1/,$+ n f f^{\text {c00617 }}$ flies, as shown in Figures 5-7. Control flies were $w(C S 10)$. For $24 \mathrm{~h}$ memory, $n=14$. For all other data, $n=6$. Means \pm SEM are shown. ${ }^{*} p<0.05,{ }^{* *} p<0.001$ relative to controls.
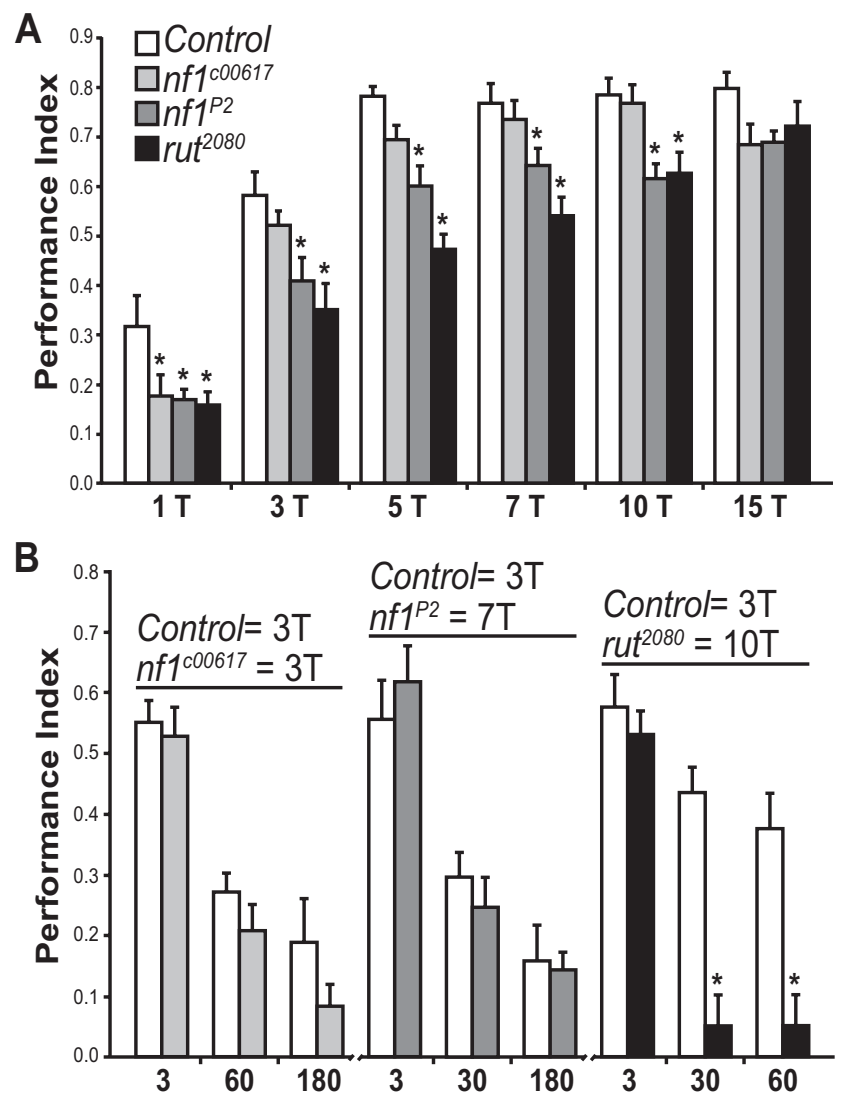

Figure 2. Neurofibromin is required for memory acquisition, not memory stability. $\boldsymbol{A}, \mathrm{nf1}$ mutants are defective in the acquisition of memories. All groups were trained with 1-15 trials $(\mathrm{T})$, and performance was assayed immediately after conditioning. With one training trial exposure, all mutants performed significantly poorer than controls. When exposed to 15 training trials, all mutants performed at the same level as controls. For all other experiments, both $n f 1^{P 2}$ and rut ${ }^{2080}$ mutants performed significantly poorer than controls, whereas $n f 1^{\text {c00617 }}$ mutants did not. Across experiments, the performance of $n f 1^{P 2}$ and rut ${ }^{2080}$ mutants paralleled that of controls but was delayed. Control flies were $w(\mathrm{CS} 10)$ for all experiments. For the five trial experiment, $n=9$ for each group. For all other experiments, $n=6$ for each group. Means \pm SEM are shown. ${ }^{*} p<0.05$. B, Memory stability is normal in $n f 1$ mutants but defective in rut ${ }^{2080}$ mutants. Mutant performance at 3 min after training was normalized to control performance by varying the number of training trials, as indicated. Performance of $n f 1$ homozygous mutants was not significantly different from control performance at the time points tested. Whereas rut $^{2080}$ and control performances were indistinguishable 3 min after training, they were significantly different at both 30 and 60 min after training. Control flies were $w$ (CS10) for all experiments. For 30 min decay of $n f 1^{P 2}$ performance, $n=11$. For all other experiments, $n=6$ for each group. Means \pm SEM are shown. ${ }^{*} p<0.005$.

ceiling level with as few as five training trials. After a single training trial, all mutants performed poorly relative to the control, but $n f 1^{\text {c00617 }}$ flies did not continue to display a learning deficit when presented with additional training trials. In contrast, $n f 1^{P 2}$ and rut $^{2080}$ flies performed poorly until after 15 training trials, at which point their performance was not significantly different from the control. Our results suggest that both Nf1 and Rut-AC are required for the acquisition of olfactory memory.

Immediate performance of each mutant was then normalized relative to the control so that memory decay could be compared (Fig. $2 \mathrm{~B}$ ). When $n f 1^{c 00617}$ mutants and controls were both trained with three trials, immediate memory was identical. Likewise, memory tested at two subsequent time points was indistinguishable. Normalization of $n f 1^{P 2}$ performance required training with seven trials when the control was trained with three trials. Memory of $n f 1^{P 2}$ mutants, when tested at two subsequent time points, 
was also indistinguishable from performance of controls, suggesting that Nf1 is not required to maintain olfactory memories over time. Finally, the immediate performance of $\mathrm{rut}^{2080}$ and control flies was normalized by 10 and 3 training trials, respectively. As early as $30 \mathrm{~min}$ after training, the memory of rut ${ }^{2080}$ flies was abolished, suggesting that Rut-AC plays an important role in maintaining memories over time. Furthermore, our results suggest that the role of Rut-AC in memory stability is Nf1 independent.

\section{$n f 1$ is expressed broadly in the brain and in mushroom body neurons}

Walker et al. (2006) reported widespread expression of Nf1 in the CNS of Drosophila larvae but a notable absence of expression in third-instar mushroom body neurons. Additionally, the authors also indicated an absence of Nf1 expression in the adult mushroom body neurons but expression elsewhere in the adult CNS. However, the putative association of Nf1 with Rut-AC in learning (Guo et al., 2000), the preferential expression of Rut-AC in mushroom body neurons (Han et al., 1992), and the clear evidence indicating that Rut-AC is required in adult mushroom body neurons for olfactory learning (Zars et al., 2000; McGuire et al., 2003; Mao et al., 2004; Akalal et al., 2006; Blum et al., 2009) necessitates Nf1 expression in adult mushroom body neurons.

We therefore probed $n f 1$ gene expression by RNA in situ hybridization on $w(C S 10)$ brain sections. Figure 3 illustrates that $n f 1$ is expressed in many, or all, cell body regions of the central brain. Expression of $n f 1$ was apparent in cell body regions surrounding the antennal lobes, protocerebrum, lateral horn, and mushroom body calyces. Additional examination revealed $n f 1$ RNA expression in many mushroom body cell bodies (Fig. $3 E$ ). Hybridization of the same probe with $n f 1^{P 1}$ homozygous mutant brains did not result in staining, confirming the specificity of the antisense probe. Our results indicate that the $n f 1$ gene is broadly expressed in the adult CNS, including the mushroom body neurons.

Considering the robust expression of $n f 1$ RNA in mushroom body cell bodies, it is surprising that attempts to visualize the Nf1 protein in adult mushroom body neurons, with the same antibodies used for larval immunohistochemistry, have not been successful (Walker et al., 2006) (our unpublished observations). However, three different Nf1 isoforms exist, which encode peptides of 2746 (Nf1-RC), 2764 (Nf1-RD), and 2802 (Nf1-RB) amino acids. It is not known which of these is expressed in the adult CNS. We have developed RNA probes against a sequence region that is common among all transcripts. Walker et al. (2006) used monoclonal antibodies (DNF1-21) generated against the C-terminal 450 amino acids of Nf1-RB (The et al., 1997). Only 195 of these residues are present in Nf1-RC, and these are followed by 199 resides that are not in common with Nf1-RB. Furthermore, only 411 of the 450 amino acids in Nf1-RB are present in Nf1-RD, and this is followed by one residue that is not in common with Nf1-RB. It seems probable that the DNF1-21 does not react with the isoform that is expressed in mushroom body neurons.

Many other genes, required for learning and memory, are preferentially expressed in mushroom body neurons (Nighorn et al., 1991; Han et al., 1992; Skoulakis et al., 1993; Cheng et al., 2001; Davis, 2005), so expression of Nf1 in the adult mushroom bodies makes sense. Additionally, the broad nature of $n f 1$ expression in the central brain is not surprising because of its role in other behavioral outputs, i.e., circadian rhythm (Williams et al., 2001) and escape latency (The et al., 1997), both of which may require $\mathrm{Nf1}$ expression in distinct neurons of the adult brain.

\section{$n f 1$ expression in mushroom body neurons, only during adulthood, rescues $3 \mathrm{~h}$ memory}

Previous work using a ubiquitously expressed, heat-shockinducible, wild-type transgene ( $h s n f 1)$ demonstrated that $n f 1$ is required in adulthood for normal olfactory associative learning (Guo et al., 2000). Although these experiments identified a physiological role for Nf1 in memory formation, they did not identify the neurons that require Nf1 for normal olfactory learning. After this work was completed, the Gene-Switch system was developed in our laboratory for simultaneous temporal and spatial control of gene expression (Mao et al., 2004). Gene-Switch is a Gal4based, RU486-inducible regulator of uas transgene expression. With this system, we have been able to simultaneously identify 

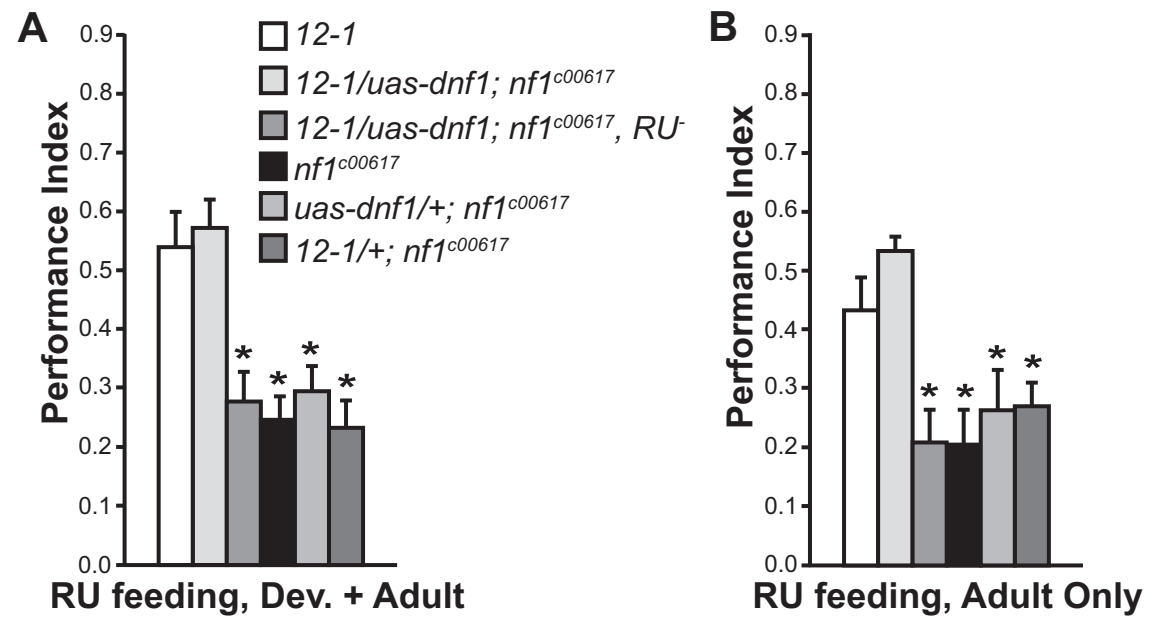

Figure 4. nf1 expression in mushroom body neurons, only during adulthood, rescues $n f 7^{\text {c00617 }}$ memory. $n f 1$ expression was restored in mushroom body neurons of homozygous $n f 1^{\text {c00617 }}$ mutants by expressing uas- dnf1 under the control of the Gene-Switch system. $A$, All groups were fed RU486 during development and adulthood, except as indicated, and performance was assayed $3 \mathrm{~h}$ after training. Expression of $n f 1$ during development and adulthood resulted in complete rescue of the $3 \mathrm{~h}$ memory phenotype. For all groups, $n=6$. Means \pm SEM are shown. ${ }^{*} p<0.005$. $B$, All groups were fed standard food during development and were transferred to food containing RU486 for $5 \mathrm{~d}$ after eclosion, except as indicated. Performance was assayed $3 \mathrm{~h}$ after training. Expression of $n f 1$, only during adulthood, completely rescued the $3 \mathrm{~h}$ memory phenotype. For all groups, $n=6$. Means \pm SEM are shown. ${ }^{*} p<0.05$.
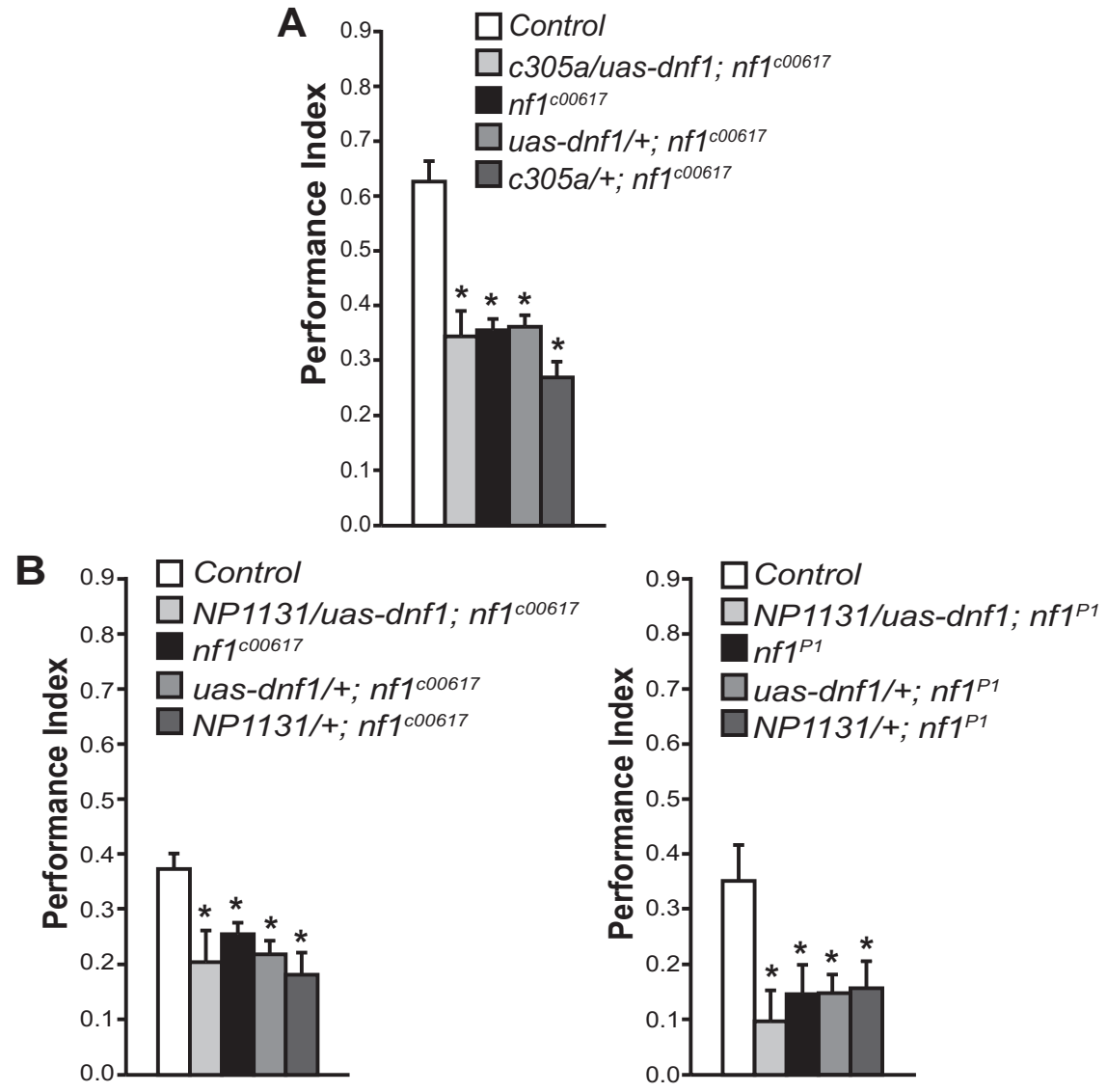

Figure 5. $n f 1$ expression in $\alpha^{\prime} / \beta^{\prime}$ or $\gamma$ neurons does not restore $3 \mathrm{~h}$ memory. $\boldsymbol{A}$, Expression of $n f 1$ was restored in $\alpha^{\prime} / \beta^{\prime}$ neurons of homozygous $n f f^{000617}$ mutants by expressing a uas-dnf1 transgene under control of the $305 a-g a l 4$ driver. Performance was assayed $3 \mathrm{~h}$ after training. K33 was used as the control. All genotypes showed significant memory impairment relative to control flies. For all groups, $n=6$. Means \pm SEM are shown. ${ }^{*} p<0.0001$. $\boldsymbol{B}$, Expression of $n f 1$ was restored in $\gamma$ neurons of homozygous $n f 1^{\mathrm{C00617}}$ or $n f 1^{P 1}$ mutants by expressing uas - dnf1 under the control of the NP1131-gal4 driver. Performance was assayed $3 \mathrm{~h}$ after training. Control flies were $w(C S 10)$. All genotypes show significant memory impairment relative to control flies. For all groups, $n=6$. Means \pm SEM are shown. ${ }^{*} p<0.05$. time and space requirements of $n f 1$ expression for normal olfactory learning.

Expression of uas-dnf1 was first induced by Gene-Switch line 12-1 during both development and adulthood, to confirm that this system could induce sufficient $n f 1$ expression to rescue the $n f 1^{\text {c00617 }}$ $3 \mathrm{~h}$ memory phenotype. Gene-Switch line 12-1 uses a mushroom body enhancer that induces expression in both $\alpha / \beta$ and $\gamma$ mushroom body neurons (Mao et al., 2004), and performance of this line does not differ from $w($ CS10) controls (data not shown). Animals were reared on food laced with RU486 from embryogenesis, through training and testing as adults. Expression of uas-dnfl in mushroom body neurons during development and adulthood resulted in complete rescue of the 3-h memory phenotype (Fig. 4A). Next, flies were reared on normal food and transferred to food containing RU486 at eclosion. Our results indicate that expression of $n f 1$, only in adult mushroom body neurons, is sufficient for complete rescue of the $n f 13 \mathrm{~h}$ memory phenotype (Fig. 4B).

\section{$n f 1$ expression in $\boldsymbol{\alpha}^{\prime} / \boldsymbol{\beta}^{\prime}$ or $\boldsymbol{\gamma}$ neurons does not restore $3 \mathrm{~h}$ memory}

Recent efforts, in our laboratory and others, have begun to distinguish the specific roles of individual mushroom body neuron subtypes in learning and memory $(\mathrm{Yu}$ et al., 2005, 2006; Akalal et al., 2006; Krashes et al., 2007). Some evidence suggests that synaptic output from $\alpha^{\prime} / \beta^{\prime}$ neurons is required during acquisition and consolidation for the formation of stable memories (Krashes et al., 2007; Wang et al., 2008). Because our data implies a role for neurofibromin in memory acquisition, we decided to see whether $n f 1$ expression in $\alpha^{\prime} / \beta^{\prime}$ neurons would be sufficient to rescue the $3 \mathrm{~h}$ memory phenotype.

$n f 1$ was expressed in $\alpha^{\prime} / \beta^{\prime}$ neurons of $n f^{100617}$ flies with the $c 305 a-$ gal4 driver (Krashes et al., 2007; Aso et al., 2009). It was not possible to test for rescue of the $n f 1^{P 1}$ phenotype, because $w ; c 305 a ; n f 1^{P 1}$ animals have severely reduced fecundity. However, expression of $n f 1$ in this subset of mushroom body neurons was not sufficient to rescue the $3 \mathrm{~h}$ memory phenotype of $n f 1^{c 00617}$ homozygous mutants (Fig. 5A). This result suggests that Nf1dependent signaling pathways are not required in $\alpha^{\prime} / \beta^{\prime}$ neurons for acquisition and early memory formation.

A close association between $n f 1$ and rut- $A C$ during learning has been well established (Guo et al., 2000; Ho et al., 2007), and expression of rut-AC in $\gamma$ neu- 
rons of the mushroom bodies partially rescues the learning phenotype of rut mutants (Zars et al., 2000; McGuire et al., 2003; Mao et al., 2004; Akalal et al., 2006; Blum et al., 2009). Therefore, we examined whether uas-dnf1 expression in $\gamma$ neurons might rescue the phenotype of nf1 mutants. Driver NP1131-gal4 was used to express uas-dnf1 in $\gamma$ neurons (Akalal et al., 2006; Aso et al., 2009). However, expression of $n f 1$ in this subset of mushroom body neurons was not sufficient to rescue the $3 \mathrm{~h}$ memory phenotype of either mutant allele (Fig. $5 B$ ). This result suggests that Nf1-dependent signaling pathways are not required in $\gamma$ neurons during memory acquisition and early consolidation. Furthermore, any role of Rut-AC in $\gamma$ neurons, during PSI-EM processing, is $\mathrm{Nf1}$ independent.

\section{$n f 1$ expression in $\alpha / \beta$ neurons restores} protein synthesis-independent memory Because expression of rut-AC in $\alpha / \beta$ neurons partially rescues the learning phenotype of rut mutants (Zars et al., 2000; McGuire et al., 2003; Mao et al., 2004; Akalal et al., 2006; Blum et al., 2009), we next expressed uas-dnf1 in both $n f 1^{P 1}$ and

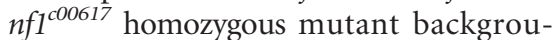
nds using the Mz1081-gal4 driver. With this driver, transgene expression is promoted throughout the central brain, including antennal lobe and $\alpha / \beta$ neurons. Expression of uas $-d n f 1$ in these neurons was sufficient to rescue the $3 \mathrm{~h}$ memory phenotype of both $n f 1$ homozygous mutant alleles (Fig. 6A). Next, we used the NP9-gal4 driver, which shows a more limited expression pattern relative to Mz1081-gal4. NP9 is also broadly expressed in the central brain, including antennal lobe and $\alpha / \beta$ neurons. Expression of uas-dnf1 with this driver was also able to fully rescue the $3 \mathrm{~h}$ memory phenotype of both $n f 1^{P 1}$ and $n f 1^{c 00617}$ homozygous mutant alleles (Fig. 6B). We next expressed uas-dnf1 using the c739-gal4 driver, which promotes robust transgene expression in the $\alpha / \beta$ mushroom body neurons and weak transgene expression in other neurons of the central brain (Aso et al., 2009). Once again, we saw complete rescue of the $3 \mathrm{~h}$ memory phenotype, providing more substantial evidence that the expression of $n f 1$ only in $\alpha / \beta$ neurons is sufficient for restoring normal memory in nf1 mutants (Fig. 6C). Finally, we expressed uas-dnf1 using $17 d-$ gal4, which promotes transgene expression in the central core of $\alpha / \beta$ mushroom body neurons (Aso et al., 2009). Expression with this driver did not restore normal $3 \mathrm{~h}$ memory of either mutant allele (Fig. $6 D)$, suggesting that either the expression level induced by this
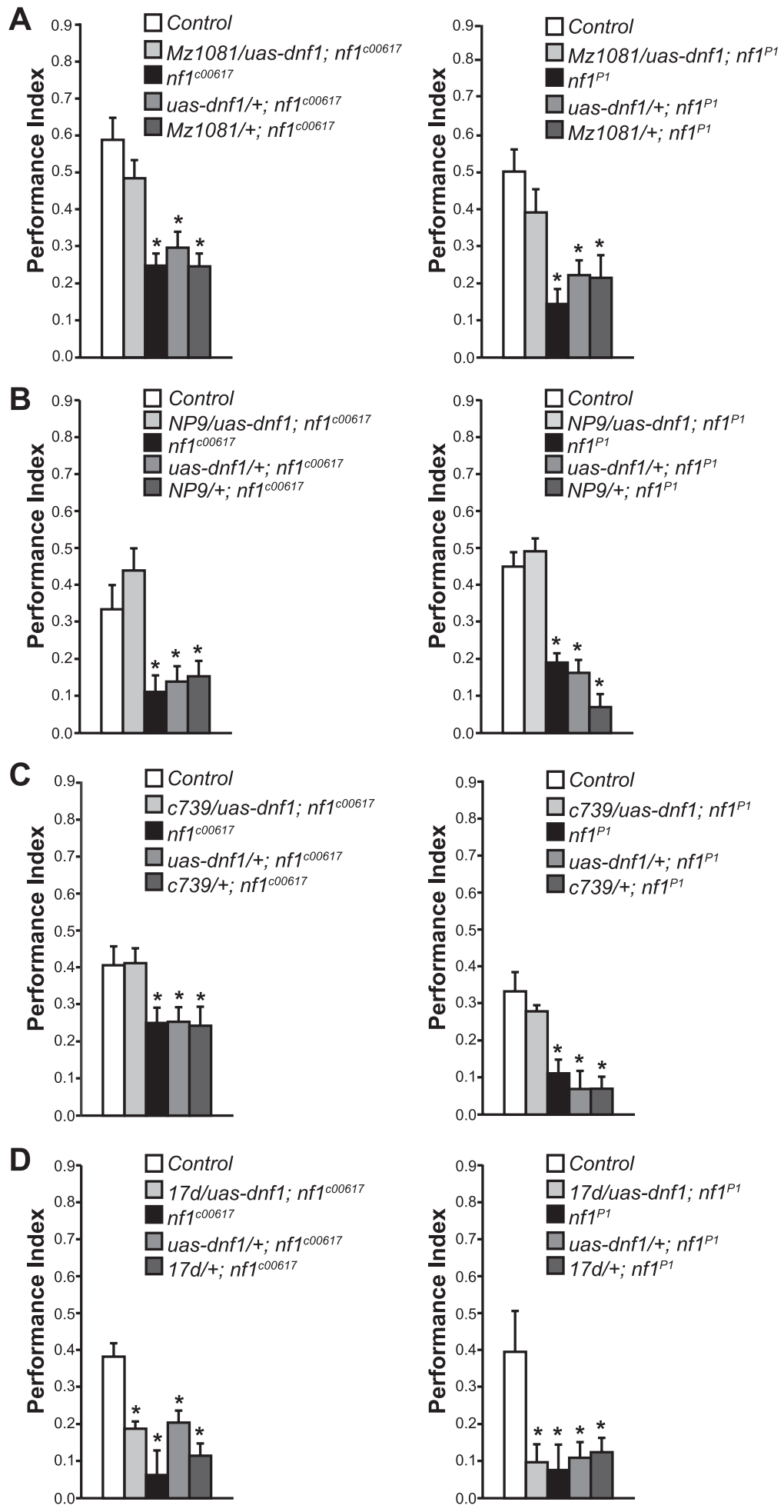

Figure 6. $\quad n f 1$ expression in $\alpha / \beta$ neurons rescues $3 \mathrm{hmmemory}$. Expression of $n f 1$ was restored in $\alpha / \beta$ neurons of homozygous $n f 7^{000617}$ and $n f 7^{P 1}$ mutants by expressing a uas - dnf1 transgene under the control of either Mz1081-gal4 (A), NP9 - gal4 (B), 7339 - gal4 (C), or $17 d-g a / 4$ (D). Performance was assayed $3 \mathrm{~h}$ after training. $A-C$, Expression of $n f 1$ in $\alpha / \beta$ neurons resulted in complete $3 \mathrm{~h}$ memory rescue of both alleles. K33 was used as the control. All negative control groups showed significant memory impairment relative to control flies. D, Expression of $n f 1$ under control of $17 d$ - gal 4 failed to rescue $3 \mathrm{~h}$ memory. Control flies were $w$ (CS10). All genotypes showed significant memory impairment relative to control flies. For all experiments, $n=5-9$. Means \pm SEM are shown. ${ }^{*} p<0.05$.

driver is not sufficient or that it does not promote expression in a sufficient number of $\alpha / \beta$ neurons to support learning.

We also tested whether uas-dnf1 expression in $\alpha / \beta$ neurons would rescue 3 min memory, the earliest testable form of PSI- 

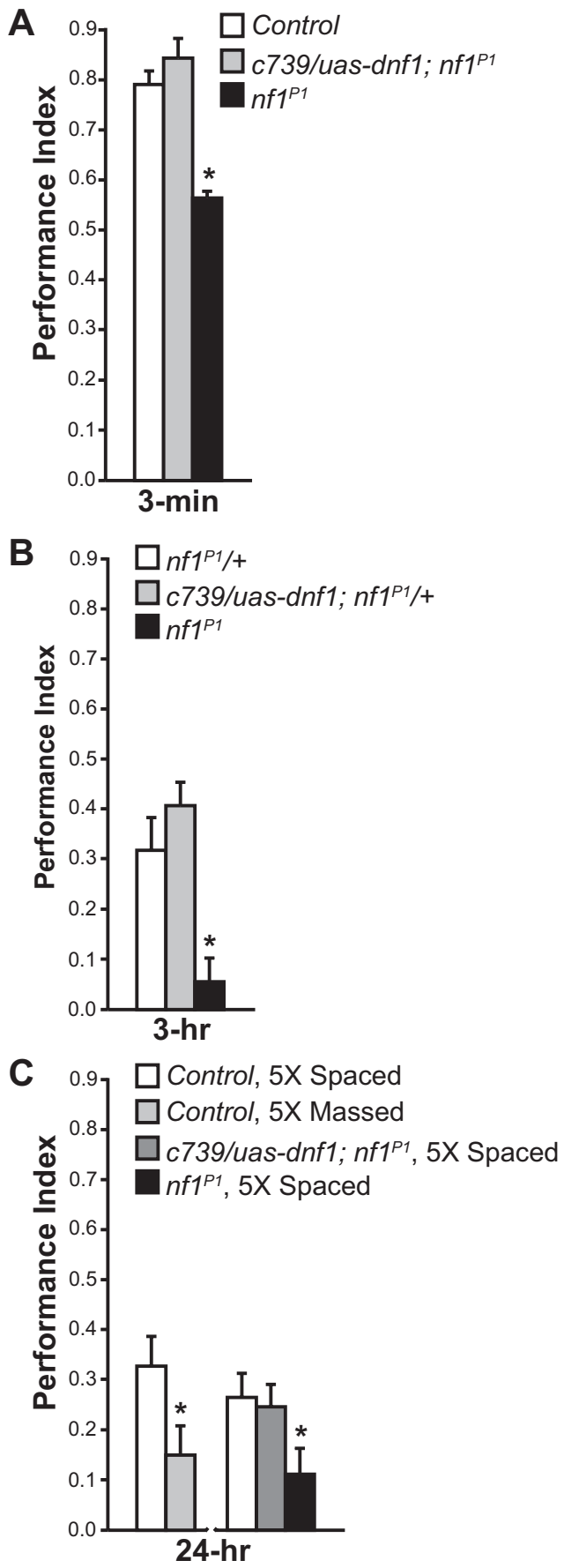

Figure 7. $n f 1$ expression in $\alpha / \beta$ neurons also rescues $3 \mathrm{~min}$ and $24 \mathrm{~h}$ memory but does not enhance control performance. $\boldsymbol{A}$, Three minute memory was rescued by $n f 1$ expression in $\alpha / \beta$ neurons. Expression of $n f 1$ was restored in $\alpha / \beta$ neurons of homozygous $n f 1^{P 1}$ mutants by expressing a uas - dnf1 transgene under control of the 739 - gal 4 driver, and performance was assayed 3 min after training. Complete rescue of the phenotype was observed, consistent with experiments showing rescue of $3 \mathrm{~h}$ memory. Control flies were $w$ (CS10). For all groups, $n=6$. Means \pm SEM are shown. ${ }^{*} p<0.005 . \boldsymbol{B}, n f 1$ expression in $\alpha / \beta$ neurons rescued $24 \mathrm{~h}$ memory. Expression of $n f 1$ was restored in $\alpha / \beta$ neurons of homozygous $n f f^{P 1}$ mutants by expressing a uas-dnf1 transgene under control of the c739-gal4 driver. Performance was assayed $24 \mathrm{~h}$ after $5 \times$-spaced training, which produced long-lasting memory that was significantly different from that elicited by $5 \times$-massed training. K33 was used as the control. For spaced versus massed, $n=12$. For rescue, $n=15$. Means \pm SEM are shown. ${ }^{*} p<0.05$. C, Control performance is not affected by neurofibromin overexpression in $\alpha / \beta$ neurons. Overexpression of uas- $d n f 1$ in $\alpha / \beta$ neurons of heterozygous $n f 1^{P 1}$ flies was driven by c739-gal4, and performance was assayed $3 \mathrm{~h}$ after training. For all groups, $n=5$. Means \pm SEM are shown. ${ }^{*} p<0.005$.
EM. We used the $c 739-$ gal4 driver to promote expression of uas-dnf1 in $\alpha / \beta$ neurons of $n f 1^{P 1}$ homozygous mutant flies. We observed full rescue of the $n f 1$ phenotype at 3 min after training (Fig. 7A). Additionally, we tested the effect of overexpressing $n f 1$ in normally performing flies using $c 739$-gal4. Consistent with a previous report (Guo et al., 2000), we observed no enhancement of normal performance when $n f 1$ was overexpressed in $\alpha / \beta$ neurons (Fig. 7B).

Because Mz1081-gal4, NP9-gal4, and c739-gal4 drivers all promote some expression outside of the $\alpha / \beta$ neurons, i.e., in antennal lobe neurons, we wanted to further demonstrate that expression of $n f 1$ in $\alpha / \beta$ neurons, rather than these other sites of expression, rescued the memory phenotype. Our laboratory previously introduced an $M B\{G a l 80\}$ transgene into the chromosome carrying c772-gal4, by recombination (Liu et al., 2007). The $M B\{$ Gal80\} transgene contains the gal80 repressor gene for Gal4 (Lee and Luo, 1999) downstream of a mushroom body enhancer, which drives expression predominantly in the mushroom body neurons (Zars et al., 2000; Mao et al., 2004). Combining the $M B\{G a l 80\}$ transgene with $c 772-$ gal4 specifically suppresses uas transgene expression in the mushroom body neurons, whereas expression in antennal lobe neurons is unaffected (Liu et al., 2007). Expression promoted by $c 772-$ gal4 by itself is strongest in $\alpha / \beta$ and $\gamma$ mushroom body neurons, moderate in antennal lobe neurons, and weak in $\alpha^{\prime} / \beta^{\prime}$ mushroom body neurons (Liu et al., 2007; Aso et al., 2009).

As expected, the $n f 1^{c 00617} 3 \mathrm{~h}$ memory phenotype was fully rescued when $c 772-$ gal4 was used to express uas-dnf1 (Fig. 8A). However, flies carrying the combined c772-gal4; $M B\{$ Gals0\} driver along with uas-dnf1 exhibited a performance score that was indistinguishable from $n f 1^{c 00617}$ homozygous mutants (Fig. $8 B$ ). Therefore, rescue of the $3 \mathrm{~h}$ memory phenotype of $n f 1 \mathrm{mu}-$ tants requires expression in $\alpha / \beta$ mushroom body neurons.

\section{$n f 1$ expression in $\alpha / \boldsymbol{\beta}$ neurons restores protein} synthesis-dependent long-term memory

Recent work in our laboratory suggests that a memory trace can be visualized in the $\alpha / \beta$ neurons of mushroom bodies $24 \mathrm{~h}$ after adult flies are subjected to a $5 \times$-spaced training procedure (see Materials and Methods) that produces PSD-LTM (Yu et al., 2006). In contrast, a $5 \times$-massed training procedure produces neither PSD-LTM nor a memory trace in $\alpha / \beta$ neurons. A deficit in LTM has been reported for $n f 1$ mutants trained with a spaced protocol (Ho et al., 2007). It is possible that this phenotype is attributable to the requirement for $n f 1$ in $\alpha / \beta$ neurons. We therefore used c739-gal4 to promote expression of uas-dnf1 in $\alpha / \beta$ neurons of $n f 1^{P 1}$ homozygous mutant flies and tested for rescue $24 \mathrm{~h}$ after $5 \times$-spaced training. We observed full rescue of the $n f 1$ phenotype at $24 \mathrm{~h}$ (Fig. 7C). Because expression of $n f 1$ in $\alpha / \beta$ neurons can restore both PSI-EM and PSD-LTM, our results suggest that Nf1 mediates multiple types of memory processing, through at least two different biochemical pathways, within the same population of neurons.

\section{Discussion}

Regardless of species being studied, neurofibromin is involved in many different brain activities, including, but not limited to, cognitive processes, circadian rhythms, cortical development, and glial development. Even within the cognitive realm, Nf1 function depends on the context of specific training conditions. Protein synthesis-independent short- and middle-term memories appear to require an activation of Rut-AC by Nf1, whereas protein synthesis-dependent long-term memory requires an additional 

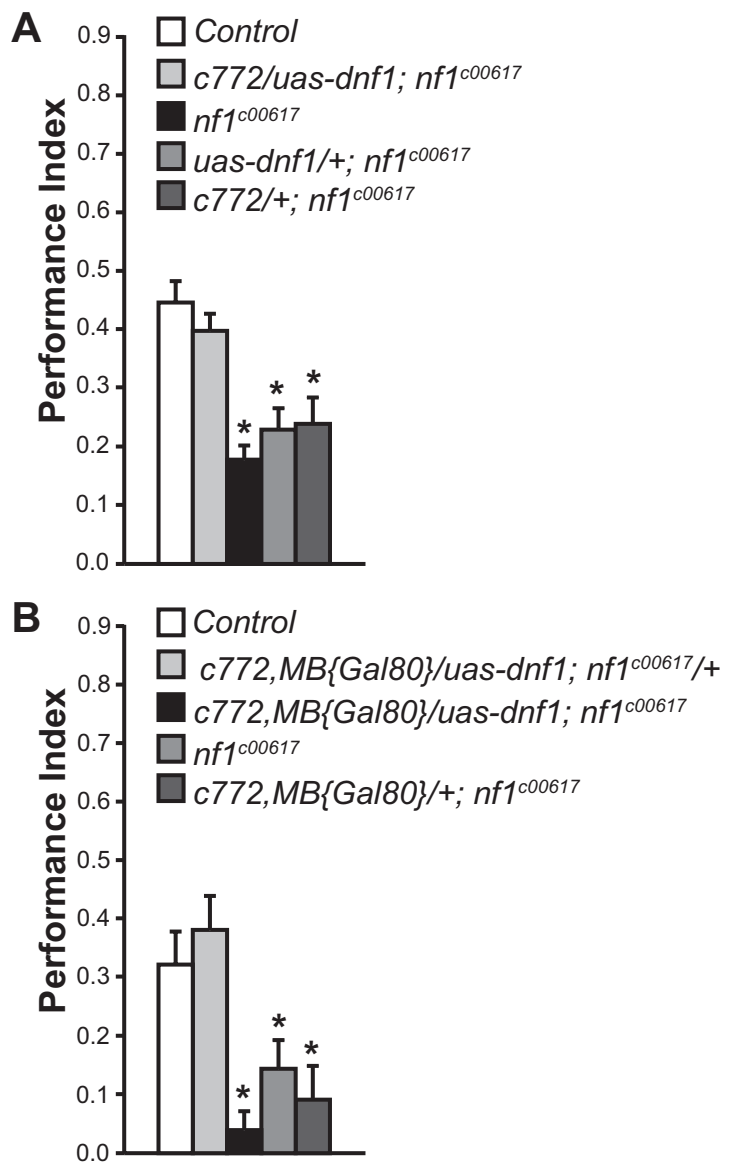

Figure 8. Inhibiting $n f 1$ expression in $\alpha / \beta$ neurons prevents memory rescue. $A$, Expression of $n f 1$ was restored in $\alpha / \beta$ neurons of homozygous $n f 1^{\text {c00617 }}$ mutants by expressing a uasdnf1 transgene under control of the $7772-$ gal4 driver. No significant difference was observed between controls and c772/uas- dnf1; $n f 1^{\text {c00617 }}$ flies, but all other genotypes showed a significant memory impairment relative to controls. Control flies were $w$ (CS10). Performance was assayed $3 \mathrm{~h}$ after training. For all groups, $n=15$. Means \pm SEM are shown. ${ }^{*} p<0.005$. $\boldsymbol{B}$, When $M B\{G a / 80\}$ was introduced to inhibit Gal4 activity in mushroom body neurons, expression of $n f 1$ by 7772 gal 4 did not rescue the $3 \mathrm{~h}$ memory phenotype of $n f 1^{\text {c00617 }}$. Similar expression of uas-dnf1 in a heterozygous $n f 1^{\text {c00617 }}$ genetic background did not alter performance relative to control, but all other genotypes showed significant memory impairment by comparison. Performance was assayed $3 \mathrm{~h}$ after training. Control flies were $w$ (CS10). For all groups, $n=$ 8. Means \pm SEM are shown. ${ }^{*} p<0.05$.

modulation of Ras activity (Hannan et al., 2006; Ho et al., 2007). By rescuing the performance of homozygous mutants, we have demonstrated that expression of Nf1 in adult $\alpha / \beta$ mushroom body neurons is sufficient to support all forms of Nf1-dependent memory. We have also revealed a requirement for Nf1 during acquisition. Together, our observations expand our current understanding of Nf1 and Rut-AC functions and challenge current models of mushroom body neuron activity in olfactory memory formation.

Rut-AC is required in both $\alpha / \beta$ and $\gamma$ mushroom body neurons for complete rescue of rut STM deficits (Akalal et al., 2006), yet we have shown that Nf1 is required only in the $\alpha / \beta$ mushroom body neurons. It is unclear why Rut-AC activation would only require Nf1 in one subset of neurons. One possibility is that the Nf1 stimulation of Rut-AC in $\alpha / \beta$ neurons during acquisition may indirectly facilitate, through unknown signals, Rut-AC activity in the $\gamma$ neurons. Previous results have been interpreted to suggest that there are communication loops that exist between certain types of mushroom body neurons and with extrinsic mushroom body neurons for normal learning and consolidation (Yu et al., 2006; Krashes et al., 2007). A similar process could allow Rut-AC activation in $\gamma$ neurons to be indirectly dependent on Nf1 in $\alpha / \beta$ mushroom body neurons. Alternatively, it could be that the Rut-AC is dependent on Nf1 in the $\alpha / \beta$ mushroom body neurons for its role in learning but Nf1 independent in $\gamma$ mushroom body neurons.

For rescue of PSD-LTM, both Rut-AC (Blum et al., 2009) and $\mathrm{Nf1}$ expression are required only in $\alpha / \beta$ neurons, suggesting that their interaction is necessary to support this form of memory as well. A recent study concluded that the Nf1-GRD, which has been shown to mediate an adenylyl cyclase activity (Hannan et al., 2006), is necessary and sufficient for Nf1-dependent LTM (Ho et al., 2007). In contrast to Rut-AC, this adenylyl cyclase activity is stimulated by Ras and is $\mathrm{G} \alpha_{\mathrm{s}}$ independent. It is important to note, however, that the Nf1-GRD domain only partially rescued the LTM phenotype of $n f 1$ mutants. Full rescue of LTM required a full-length $n f 1$ transgene. Together, these data and ours suggest that Nf1 simultaneously mediates the activation of both AC signaling pathways in $\alpha / \beta$ neurons to facilitate new protein synthesis and the formation of long-lasting memory.

Early work on the role of Rut-AC in olfactory associative memory suggested that this adenylyl cyclase plays a role in behavioral acquisition (Tully and Quinn 1985; Dudai et al., 1988). Using an olfactory avoidance assay, it was suggested that rutabaga mutants could obtain normal performance with more intense training (Dudai et al., 1984). We have also observed a delay in the acquisition of olfactory memory in rutabaga mutants, which require three times the amount of training as controls to overcome. A similar delay in acquisition was discovered for $n f 1$ mutants, consistent with the hypothesis that Nf1 is required for G-protein activation of Rut-AC during learning (Guo et al., 2000; Ho et al., 2007). Our results also demonstrate that Rut-AC is essential for the stability of olfactory memory. However, this function is independent of an interaction with Nf1. We believe that the association of Nf1 and Rut-AC may be transient, only required for the initial activation of Rut-AC in its role as a molecular coincidence detector in $\alpha / \beta$ neurons (Tomchik and Davis, 2009). If this model were true, memory stability would therefore require continued stimulation of Rut-AC molecules via an independent and perhaps spatially distinct mechanism that does not require Nf1.

Recent efforts, in our laboratory and others, have attempted to assign temporal and operational phases of olfactory memory processing to distinct regions within the adult olfactory system. During pairing of odor and electric shock, new projection neuron synapses are recruited to the odor representation (Yu et al., 2004). Pairing dopamine application with neuronal depolarization in adult brain preparations results in a Rut-AC-dependent synergistic increase of cAMP in both $\alpha$ and $\alpha^{\prime}$ lobes (Tomchik and Davis, 2009), and memory acquisition requires synaptic transmission from $\alpha^{\prime} / \beta^{\prime}$ neurons (Krashes et al., 2007). Although we have demonstrated that both Nf1 and Rut-AC are required for memory acquisition, neither of these need be expressed in $\alpha^{\prime} / \beta^{\prime}$ neurons (Zars et al., 2000; McGuire et al., 2003; Mao et al., 2004; Akalal et al., 2006; Blum et al., 2009). We therefore propose that memory acquisition cannot be thought of as a specific event involving a distinct neuronal subset. Rather, we envision a model in which the pairing of odor and electric shock induces a change on the neuronal systems level. Each individual neuron subset may register this change in a different way, but every change is in some way necessary for memory acquisition as a whole. Additional work will be required to determine whether memory consolida- 
tion, retrieval, or processing of longer-term memories also require plasticity throughout the entire olfactory system.

It is clear from the data herein that Nf1 function is required in the adult brain, in $\alpha / \beta$ neurons defined by the $c 739-$ gal4 driver, for PSI-EM formation and for PSD-LTM formation. By identifying a minimal region in which Nf1 expression is required, we are now able to isolate its role in memory formation from others that may occur in the brain. This mapping promises a more accurate analysis of Nf1-dependent memory and insights into both memory processing as a whole and into the cognitive deficits associated with neurofibromatosis type 1 .

\section{References}

Acosta MT, Gioia GA, Silva AJ (2006) Neurofibromatosis type 1: new insights in neurocognitive issues. Curr Neurol Neurosci Rep 6:136-143.

Akalal DB, Wilson CF, Zong L, Tanaka NK, Ito K, Davis RL (2006) Roles for Drosophila mushroom body neurons in olfactory learning and memory. Learn Mem 13:659-668.

Aso Y, Grübel K, Busch S, Friedrich AB, Siwanowicz I, Tanimoto H (2009) The mushroom body of adult Drosophila characterized by GAL4 drivers. J Neurogenet 23:156-172.

Beck CD, Schroeder B, Davis RL (2000) Learning performance of normal and mutant Drosophila after repeated conditioning trials with discrete stimuli. J Neurosci 20:2944-2953.

Bernards A, Snijders AJ, Hannigan GE, Murthy AE, Gusella JF (1993) Mouse neurofibromatosis type $1 \mathrm{cDNA}$ sequence reveals high degree of conservation of both coding and non-coding RNA segments. Hum Mol Genet 2:645-650.

Blum AL, Li W, Cressy M, Dubnau J (2009) Short-and long-term memory in Drosophila require cAMP signaling in distinct neuron types. Curr Biol 19:1341-1350.

Cheng Y, Endo K, Wu K, Rodan AR, Heberlein U, Davis RL (2001) Drosophila fasciclinII is required for the formation of odor memories and for normal sensitivity to alcohol. Cell 105:757-768.

Davis RL (2005) Olfactory memory formation in Drosophila: from molecular to systems neuroscience. Annu Rev Neurosci 28:275-302.

Dudai Y, Zvi S, Segel S (1984) A defective conditioned behavior and a defective adenylate cyclase in the Drosophila mutant rutabaga. J Comp Physiol A Neuroethol Sens Neural Behav Physiol 155:569-576.

Dudai Y, Corfas G, Hazvi S (1988) What is the possible contribution of $\mathrm{Ca}^{2+}$-stimulated adenylate cyclase to acquisition, consolidation and retention of an associative olfactory memory in Drosophila. J Comp Physiol A Neuroethol Sens Neural Behav Physiol 162:101-109.

Guo HF, Tong J, Hannan F, Luo L, Zhong Y (2000) A neurofibromatosis1-regulated pathway is required for learning in Drosophila. Nature 403: 895-898.

Han PL, Levin LR, Reed RR, Davis RL (1992) Preferential expression of the Drosophila rutabaga gene in mushroom bodies, neural center for learning in insects. Neuron 9:619-627.

Hannan F, Ho I, Tong JJ, Zhu Y, Nurnberg P, Zhong Y (2006) Effect of neurofibromatosis type I mutations on a novel pathway for adenylyl cyclase activation requiring neurofibromin and Ras. Hum Mol Genet 15:1087-1098.

Ho IS, Hannan F, Guo HF, Hakker I, Zhong Y (2007) Distinct functional domains of neurofibromatosis type 1 regulate immediate versus longterm memory formation. J Neurosci 27:6852-6857.

Jacks T, Shih TS, Schmitt EM, Bronson RT, Bernards A, Weinberg RA (1994)
Tumour predisposition in mice heterozygous for a targeted mutation in Nf1. Nat Genet 7:353-361.

Krashes MJ, Keene AC, Leung B, Armstrong JD, Waddell S (2007) Sequential use of mushroom body neuron subsets during Drosophila odor memory processing. Neuron 53:103-115.

Lee T, Luo L (1999) Mosaic analysis with a repressible cell marker for studies of gene function in neuronal morphogenesis. Neuron 22:451-461.

Liu X, Krause WC, Davis RL (2007) GABAA receptor RDL inhibits Drosophila olfactory associative learning. Neuron 56:1090-1102.

Mao Z, Roman G, Zong L, Davis RL (2004) Pharmacogenetic rescue in time and space of the rutabaga memory impairment by using Gene-Switch. Proc Natl Acad Sci U S A 101:198-203.

McGuire SE, Le PT, Osborn AJ, Matsumoto K, Davis RL (2003) Spatiotemporal rescue of memory dysfunction in Drosophila. Science 302:1765-1768.

Nighorn A, Healy MJ, Davis RL (1991) The cAMP phosphodiesterase encoded by the Drosophila dunce gene is concentrated in mushroom body neuropil. Neuron 6:455-467.

North K (2000) Neurofibromatosis Type 1. Am J Med Genet 97:119-127.

Ozonoff S (1999) Cognitive impairment in neurofibromatosis type 1. Am J Med Genet 89:45-52.

Skoulakis EM, Kalderon D, Davis RL (1993) Preferential expression of the catalytic subunit of PKA in the mushroom bodies and its role in learning and memory. Neuron 11:197-208.

The I, Hannigan GE, Cowley GS, Reginald S, Zhong Y, Gusella JF, Hariharan IK, Bernards A (1997) Rescue of Drosophila NF1 mutant phenotype by protein kinase A. Science 276:791-794.

Thibault ST, Singer MA, Miyazaki WY, Milash B, Dompe NA, Singh CM, Buchholz R, Demsky M, Fawcett R, Francis-Lang HL, Ryner L, Cheung LM, Chong A, Erickson C, Fisher WW, Greer K, Hartouni SR, Howie E, Jakkula L, Joo D, et al. (2004) A complementary transposon tool kit for Drosophila melanogaster using P and piggyBac. Nat Genet 36:283-287.

Tomchik SM, Davis RL (2009) Dynamics of learning-related cAMP signaling and stimulus integration in the Drosophila olfactory pathway. Neuron 64:510-521.

Tully T, Quinn WG (1985) Classical conditioning and retention in normal and mutant Drosophila melanogaster. J Comp Physiol A Neuroethol Sens Neural Behav Physiol 157:263-277.

Walker JA, Tchoudakova AV, McKenney PT, Brill S, Wu D, Cowley GS, Hariharan IK, Bernards A (2006) Reduced growth of Drosophila neurofibromatosis 1 mutans reflects a non-celloautonomous requirement for GTPase-activating protein activity in larval neurons. Genes Dev 20:3311-3323.

Wang Y, Mamiya A, Chiang AS, Zhong Y (2008) Imaging of an early memory trace in the Drosophila mushroom body. J Neurosci 28:4368-4376.

Williams JA, Su HS, Bernards A, Field J, Sehgal A (2001) A circadian output in Drosophila mediated by neurofibromatosis-1 and Ras/MAPK. Science 293:2251-2256.

Yu D, Ponomarev A, Davis RL (2004) Altered representation of the spatial code for odors after olfactory classical conditioning; memory trace formation by synaptic recruitment. Neuron 42:437-449.

Yu D, Keene AC, Srivatsan A, Waddell S, Davis RL (2005) Drosophila DPM neurons form a delayed and branch-specific memory trace after olfactory classical conditioning. Cell 123:945-957.

Yu D, Akalal DB, Davis RL (2006) Drosophila alpha/beta mushroom body neurons form a branch-specific, long-term cellular memory trace after spaced olfactory conditioning. Neuron 52:845-855.

Zars T, Fischer M, Schulz R, Heisenberg M (2000) Localization of a shortterm memory in Drosophila. Science 288:672-675. 